\title{
BIOGEOGRAPHY OF INVERTEBRATES AND PLANTS IN HANGING GARDENS OF THE Colorado Plateau
}

\author{
NANCY L. STANTON $\bullet$ STEVEN J. BUSKIRK $\bullet$ JAMES F. FOWLER \\ DEPARTMENT OF ZOOLOGY AND PHYSIOLOGY \\ RONALD L. HARTMAN $\downarrow$ DEPARTMENT OF BOTANY \\ UNIVERSITY OF WYOMING $\bullet$ LARAMIE
}

\section{$\checkmark \quad$ INTRODUCTION}

The field work portion of our research on the biogeography of invertebrates and vascular plants has been completed. The geomorphological, bumble bee pollinator and plant community models developed during the first two field seasons worked well when applied to new sites in Dinosaur National Monument (DINO), Canyonlands National Park CANY), Arches National Park (ARCH), and Natural Bridges National Monument (NABR). Our research objectives for the 1993 field season were to: (1) complete the survey of plant and insect communities of hanging gardens in DINO, ARCH, CANY, and NABR; and (2) determine the geographic distribution of endemic species identified and the level of endemism within and among parks; and (3) complete the Halls Creek area survey for hanging gardens in Capitol Reef National Park (CARE).

\section{$\checkmark \quad$ RESULTS}

Over 650 plant specimens were collected in summer 1993 as vouchers for distribution and community data. These specimens are presently being identified by Dr. Hartman at the Rocky Mountain Herbarium at the University of Wyoming. Once identification is complete, analysis of community and biogeographic data on 1993 hanging gardens will begin.
Analysis of 1991-1992 plant species data sets from CARE, Glen Canyon National Recreation Area (GLCA), and Zion National Park (ZION) is continuing. The level of plant taxa endemism is different among those parks (Tables 1, 2, 3) with GLCA having a significantly higher number of endemic taxa per hanging garden than either ZION or CARE ( $F=7.674, P=.001)$. This is not an artifact of different species richness patterns since GLCA does not have significantly higher plant species richness per hanging garden (Scheffe post hoc test, GLCA vs. ZION P = .053, GLCA vs. CARE $P=.118)$. Nor does GLCA's larger number of endemic taxa appear to be related to hanging garden size among the three parks $(F=1.743, \mathrm{P}=.187)$. Community importance of endemic plant taxa as measured by their total canopy coverage has a range of $0-52 \%$ (Tables $1,2,3$ ). CARE has only one endemic hanging garden plant species, the Eastwood monkeyflower, Mimulus eastwoodiae, found only on Horseshoe Hanging Garden in small clumps (Table 3). Thus GLCA and ZION hanging gardens have a significantly higher community importance of endemic taxa than CARE $(F=5.36, P=.010)$. There is no difference between GLCA and ZION (F $=-.038, \mathrm{P}=.918)$, and both parks have large populations of endemic species on some hanging gardens. This is mainly due to large, dense patches of Rydberg's thistle, Cirsium rydbergii, in GLCA and to smaller, scattered patches of Zion shooting star, Dodecatheon pulchellum zionense, in ZION. 
Table 1. Endemism in ZION hanging garden vascular plant taxa. $R=$ species richness, $E=$ number of endemic taxa, LE = proportion of endemic taxa, CC = canopy coverage of all endemic taxa.

\begin{tabular}{|lllll|}
\hline \hline Hanging Garden & R & E & LE & CC \\
\hline ZION total & 95 & 5 & 0.05 & \\
Pine Creek & 6 & 1 & 0.17 & 0.32 \\
Upper Emerald & 33 & 2 & 0.06 & 0.23 \\
Lower Emerald & 12 & 1 & 0.08 & 0.29 \\
Grotto & 28 & 2 & 0.07 & 0.24 \\
Menu Falls & 23 & 1 & 0.04 & T \\
Fall & 14 & 1 & 0.07 & T \\
Falling Water & 21 & 2 & 0.10 & 0.22 \\
Narrows Trail & 26 & 1 & 0.04 & 0.12 \\
Trail's End & 13 & 1 & 0.08 & T \\
Canyon Overlook I & 14 & 1 & 0.07 & 0 \\
Canyon Overlook II & 26 & 1 & 0.04 & 0 \\
Court Patriarchs & 20 & 0 & 0.00 & 0.16 \\
Snail & 13 & 0 & 0.00 & 0.03 \\
Kaye's & 29 & 1 & 0.03 & NA \\
Weeping Rock & 28 & 2 & 0.07 & \\
Hailstone & 13 & 2 & 0.15 & \\
\hline
\end{tabular}

Table 2. Endemism in GLCA hanging garden vascular plant taxa.

\begin{tabular}{|c|c|c|c|c|}
\hline Hanging Garden & $\mathbf{R}$ & E & LE & $\mathrm{CC}$ \\
\hline GLCA total & 82 & 8 & 0.10 & \\
\hline Dune & 18 & 2 & 0.11 & 0.12 \\
\hline Crossbed & 22 & 3 & 0.14 & 0.18 \\
\hline Rattlesnake & 20 & 4 & 0.20 & 0.04 \\
\hline Hardwood & 29 & 3 & 0.10 & 0.01 \\
\hline Pedestal & 15 & 3 & 0.20 & 0.13 \\
\hline Zephyr & 16 & 4 & 0.25 & 0.29 \\
\hline Graffiti & 7 & 11 & 0.14 & $T$ \\
\hline Upper Three & 15 & 3 & 0.20 & 0.42 \\
\hline Lower Three & 6 & 3 & 0.50 & 0.28 \\
\hline Surprise & 9 & 0 & 0.00 & NA \\
\hline Ivy & 7 & 0 & 0.00 & NA \\
\hline Baby & 10 & 0 & 0.00 & NA \\
\hline Baby Too & 10 & 1 & 0.10 & NA \\
\hline Zigy & 30 & 7 & 0.23 & 0.43 \\
\hline Hook & 27 & 5 & 0.19 & 0.52 \\
\hline Hawk & 12 & 4 & 0.33 & 0.49 \\
\hline Swallow & 11 & 5 & 0.45 & 0.13 \\
\hline Ice & 18 & 5 & 0.28 & 0.43 \\
\hline Corner & 13 & 2 & 0.15 & 0.27 \\
\hline Channel & 11 & 4 & 0.36 & 0.27 \\
\hline Fence & 21 & 2 & 0.10 & $\mathrm{~T}$ \\
\hline Lower Cow & 14 & 2 & 0.14 & 0.15 \\
\hline Stone Basin & 16 & 0 & 0.00 & 0 \\
\hline Wrong & 11 & 0 & 0.00 & 0 \\
\hline Boondoggle & 11 & 0 & 0.00 & 0 \\
\hline Camp & 15 & 3 & 0.20 & 0.29 \\
\hline Pyro & 21 & 3 & 0.14 & $\mathrm{~T}$ \\
\hline
\end{tabular}


Table 3. Endemism in CARE hanging garden vasclar plant taxa. Legend as in Table Hanging Garden.

\begin{tabular}{|l|l|l|l|l|}
\hline \hline Hanging Garden & $\mathrm{R}$ & $\mathrm{E}$ & $\mathrm{LE}$ & $\mathrm{CC}$ \\
\hline CARE Total & 22 & 1 & 0.05 & \\
Sidewall & 12 & 0 & 0.00 & 0 \\
Horseshoe & 8 & 1 & 0.13 & $\mathrm{~T}$ \\
Meander & 3 & 0 & 0.00 & 0 \\
Beaver Dam & 9 & 0 & 0.00 & 0 \\
Fort & 4 & 0 & 0.00 & 0 \\
\hline
\end{tabular}

We collected 58 bumblebees, Bombus sp., as floral visitors on hanging gardens during the summer 1993 field season. We continued to emphasize collection from Rydberg's thistle when it was present and were able to complement our collections from GLCA by netting bumblebee floral visitors to this species in CANY and ARCH. We also collected several bumblebees from another endemic thistle, Ownbey thistle, Cirsium ownbeyii, in DINO and a small number of bumblebees from an unidentified species in NABR. These are being identified by Vince Tepidino at the USDA Bee Lab at Utah State University. General insect collections from pitfall and malaise traps are currently being sorted, prepared, and identified to family by Jim Fowler at State Fair Community College.

\section{$\checkmark \quad$ LITERATURE CITED}

Naumann, T. 1990. Inventory of Plant Species of Special Concern and the General Flora of Dinosaur National Monument 1987-89, Final Report. Unpub. ms. Colorado Natural Areas Program. Denver, CO.

Stanton, N. L., S. J. Buskirk, R. L. Hartman, and J. F. Fowler. 1993. Annual Report for Biogeography of Invertebrates of the Colorado Plateau. Unpub. ms. UW-NPS Research Center, Laramie, WY. 